\title{
Percepciones del alumnado sobre atención a la diversidad en la formación inicial del profesorado de Educación Secundaria
}

\section{Students perceptions about attention to diversity in Secondary Education Teachers Initial Training}

\author{
$\mathrm{M}^{\mathrm{a}}$ Jesús COLMENERO RUIZ ${ }^{1}$, Antonio PANTOJA VALLEJO ${ }^{1}, \mathrm{M}^{\mathrm{a}}$ del Carmen \\ PEGALAJAR PALOMINO ${ }^{2}$ \\ ${ }^{1}$ Universidad de Jaén \\ ${ }^{2}$ Universidad Católica San Antonio de Murcia
}

Recibido: Junio 2013

Aceptado: Septiembre 2013

\section{Resumen}

Con este trabajo se pretende analizar las percepciones del alumnado que cursa el Máster Universitario en Profesorado de Educación Secundaria Obligatoria, Formación Profesional y Enseñanza de Idiomas en la Universidad de Jaén, acerca de la formación inicial recibida sobre atención a la diversidad. Se sigue una metodología descriptiva que utiliza como instrumento de recogida de datos un cuestionario realizado ad hoc. Los resultados revelan las actitudes favorables de los futuros docentes de secundaria hacia la diversidad, habiendo recibido una adecuada formación en aspectos curriculares y organizativos, capaces de lograr la plena inclusión del alumnado con necesidades educativas en el aula.

Palabas clave: formación inicial, docente, atención a la diversidad, percepción, educación secundaria.

\begin{abstract}
This work aims to analyze the perceptions of students enrolled in the Master's Degree in Secondary Education Teaching, Training and Language Teaching at the University of Jaen, about the initial training received on attention to diversity. A descriptive methodology has been followed using an ad hoc questionnaire as data collection instrument. The results show favorable attitudes of future secondary teachers for diversity, having received an adequate training in curricular and organizational aspects, making it able to fully achieve inclusion of students with special educational needs in the classroom.
\end{abstract}

Keywords: initial training, teaching, attention to diversity, perceptions, secondary education.

La formación inicial del docente de Educación Secundaria constituye un motivo de preocupación en todos los países de Europa, a pesar de que en España las reformas desarrolladas no siempre han ido acompañadas de medidas adecuadas de 
transformación y mejora (Vaillant, 2009). En la actualidad, la función docente ha sufrido una transformación, de tal modo que el profesor ha debido asumir nuevas tareas y responsabilidades que le involucran en el funcionamiento del centro escolar (EURYDICE, 2008). La etapa de Educación Secundaria también ha experimentado cambios significativos en las últimas décadas a través de la obligatoriedad de la escolarización hasta los 16 años, reorganización de la formación profesional, modificación y actualización del currículum, incremento de la diversidad cultural e integración de alumnos con necesidades educativas (González, Jiménez y Pérez, 2011). Este nuevo papel para el que se tienen que preparar los futuros profesores de Educación Secundaria no sólo está marcado por una cierta intensificación de tareas (Hargreaves, 2005), sino que está provocando que el profesor vea cómo su trabajo no responde a las expectativas de aquello para lo que ha sido formado (Bolívar, 2007).

Tras la promulgación de la Ley Orgánica 2/2006, de 3 de Mayo, de Educación e inmersos en este proceso de convergencia europea en Educación Superior, la formación inicial del docente para la etapa de Educación Secundaria Obligatoria se ha organizado en torno al desarrollo de un Máster Universitario en Profesorado de Educación Secundaria Obligatoria y Bachillerato, Formación Profesional y Enseñanza de Idiomas. Dicha titulación, implantada en las universidades andaluzas desde el curso 2009/10, ha servido para paliar algunas de las deficiencias estructurales organizativas, e incluso, conceptuales que ha venido arrastrando el sistema de formación inicial del profesorado de Secundaria desde 1970 con la implantación del Curso de Aptitud Pedagógica (Imbernón, 2007). Este nuevo horizonte, definido por algunos autores como nueva oportunidad formativa para los profesores de secundaria (González Sanmamed, 2009), debe ofrecer a los futuros profesionales unos conocimientos psicopedagógicos y didácticos más profundos, a la vez que amplía el período de formación en prácticas docentes (González, Jiménez y Pérez, 2011). Además, tal y como afirman Timostsuk y Ugaste (2010), la formación inicial del profesorado de secundaria supone un espacio en el que el futuro profesor debe aprender los aspectos sociales de enseñar mediante el apoyo a la formación de la identidad docente. De este modo, dicho Máster ha sido concebido y planteado para contribuir a esta necesaria renovación de la enseñanza y no sólo para incorporar nuevos profesores al sistema educativo (Vilches y Gil-Pérez, 2010). Así pues, constituye un reto esencial mejorar la formación inicial de aquellos graduados que deciden orientar su vida laboral hacia la docencia en Educación Secundaria (Puelles, 2009), estando hasta el momento escasamente formados en materias pedagógicas, organizativas e institucionales propias de la función docente (Benso y Pereira, 2003).

Pero la finalidad principal del Máster de Secundaria no es formar profesores que sólo resuelvan las situaciones educativas, sino profesionales formados en la reflexión, resolución de problemas, investigación e innovación, que puedan contribuir a que las futuras generaciones de alumnos estén mejor preparados para afrontar los retos que se les presentan (Buendía et al., 2011). Para ello, la formación inicial del profesor de secundaria, basada en la adquisición de las competencias profesionales específicas, se debe realizar en el mismo espacio formativo y debe compartir el mismo tiempo, mediante la reflexión teórica pero también a través de la reflexión sobre la práctica (Tribó, 2008). 
El estudio realizado por Benarroch (2011) revela las valoraciones de expertos en la formación de profesores de Educación Secundaria acerca del Máster, destacando como principales debilidades, la ausencia de criterios de temporalización entre módulos así como la falta de coherencia entre los modelos de enseñanza utilizados y los que se pretenden que los futuros profesores apliquen en sus aulas. Por su parte, Reoyo, Carbonero, Freitas y Valdivieso (2012) ponen de manifiesto cómo los propios alumnos perciben la importancia de que el profesor de Educación Secundaria esté formado tanto en aspectos curriculares como afectivos y habilidades relacionales. Además, el alumnado del Máster de Secundaria no se muestra satisfecho con el tiempo establecido para su formación didáctica, la vinculación entre teoría y práctica en el desarrollo de programas formativos así como con la coordinación entre el profesorado, manifestando haberse desarrollado acciones excesivamente individualistas entre los docentes (Hernández y Carrasco, 2012).

El actual proceso de convergencia europea en Educación Superior ha mermado la formación inicial del docente para atender a la diversidad (Vela, 2010) y conseguir una enseñanza individualizada dentro del aula (Birta-Szkely, 2006); por lo que es necesaria una formación que se desarrolle desde una perspectiva más curricular y colaborativa, más cercana a la práctica, que considere como base disciplinas como la Psicología o la Pedagogía además del refuerzo educativo, las Tecnologías de la Información y la Comunicación y la formación y asesoramiento del profesorado. Algunos autores (Rodrigues y Arantes, 2007; Contreras, 2010; Korthagen, 2010; Pérez, 2010; Recchia y Puig, 2011) establecen determinadas amenazas a la formación de calidad del docente, siendo éstas: desvinculación y descontextualización del currículum de formación del docente con conocimientos mosaicales bien fragmentados por Facultades, Departamentos e incluso profesores; estudio memorístico que propicia la reflexión para que cada futuro docente construya su aprendizaje y su propio modelo educativo; divorcio entre la teoría y la práctica oficialmente establecido por las clases con carga teórica y otras con carga práctica con el error potencial de ser impartidas por profesores diferentes; separación de la experiencia y el saber; imposibilidad de llevar a efecto la investigación-acción por el divorcio también existente entre la Universidad y la escuela que agudiza el de la teoría y la práctica aunque también el de los conocimientos, habilidades, competencias, actitudes, etc.

Así pues, Sánchez (2007) revela cómo para el desarrollo de una educación de calidad para todos, no existe una adecuada formación inicial del docente para atender a la diversidad, e incluso parte de prejuicios, creencias e ideas que lo dificultan. Ello viene a reforzar la idea de Pérez (2010) para quién las instituciones formativas se encuentran lejos del ideal que supone la formación de docentes competentes, dada la necesidad de reinventar la profesión para conseguir una educación para todos (Forteza, 2011). Resultan ser los Licenciados (tanto de letras como de ciencias) los que muestran mayores carencias en su formación respecto al resto de las titulaciones de Maestro en cuestiones referidas a la atención al alumnado con necesidades educativas especiales, atención a la diversidad, alumnado con dificultades de aprendizaje, desarrollo de habilidades sociales, etc. (Camacho y Padrón, 2005). Por su parte, la investigación desarrollada por Sánchez y Boix (2008) revela cómo los futuros docentes de Educación Secundaria consideran que la heterogeneidad educativa y la diversificación pedagógica 
han de estar presentes en la formación inicial del profesorado de Secundaria; no obstante, admiten tener un desconocimiento absoluto acerca del concepto de atención a la diversidad, factores relacionados con la heterogeneidad educativa, necesidades educativas especiales, estrategias y recursos de integración e inclusión en centros y aulas ordinarias, competencias metodológicas, personales y participativas necesarias para transformar el aula y los entornos de la comunidad de aprendizaje en espacios abiertos al aprendizaje desde el diálogo.

Finalmente, algunos autores (Calvo y Susinos, 2010; Vigo, Soriano y Julve, 2010) establecen las directrices de lo que debería incluir un programa de formación inicial del profesorado en el ámbito de la Educación Especial, siendo éste: visión clara de la enseñanza a impartir, currículum fundamentado en la práctica, experiencias clínicas, estándares de la práctica, relaciones entre la universidad y la escuela, empleo de estudios de caso, indagación y evaluación y análisis reflexivo y crítico (Santos, 2010). Así pues, el docente podrá ofrecer ayudas pedagógicas adecuadas a todos los alumnos una vez cuente con elementos teóricos y prácticas que permitan favorecer el aprendizaje (Sykes, Bird y Kennedy, 2010; Conklin, 2012).

\section{Objetivos del estudio}

La finalidad de la investigación consiste en conocer las percepciones sobre atención a la diversidad del alumnado que ha cursado el Máster Universitario en profesorado de Educación Secundaria Obligatoria y Bachillerato, Formación Profesional y Enseñanza idiomas en la Universidad de Jaén durante el curso 2012/13.

De manera más específica, este trabajo pretende alcanzar los objetivos siguientes: a) elaborar un instrumento de recogida de información válido y fiable que permita obtener información acerca de las percepciones de los futuros profesores de Educación Secundaria sobre la atención a la diversidad; b) determinar el grado de relación entre los diferentes factores que conforman la escala diseñada; c) comprobar la vinculación existente entre el papel que ocupa la atención a la diversidad en la práctica docente, los conocimientos adquiridos en el máster, así como la finalidad del mismo; d) analizar si la consideración que tiene la atención a la diversidad en la práctica docente, los conocimientos adquiridos en el máster y la finalidad del mismo, tienen influencia en los factores de la escala.

En este caso, las variables que más directamente se relacionan con aspectos formativos del estudiante del Máster de Secundaria son aquellas que cuestionan si la atención a la diversidad debe ocupar un lugar importante en la futura práctica docente, así como también se pretende conocer si los estudios de Máster les han servido para adquirir suficientes conocimientos sobre Educación Especial y atención a la diversidad en el aula. Por otro lado, pretende conocer si los estudios de Máster han tenido como finalidad afianzar la elección profesional de los estudiantes hacia la docencia así como también para aumentar su interés hacia una mayor formación en el ámbito de la atención a la diversidad. Además, se han trabajado variables sociodemográficas tales como la edad, titulación de acceso y especialidad por la que cursan dichos estudios de Posgrado. 


\section{Material y métodos}

En este trabajo se ha utilizado una metodología cuantitativa basada en el método descriptivo-correlacional. Consideramos que es la adecuada cuando se desea hacer una exploración de un determinado fenómeno educativo para, a continuación, conocer esta realidad y poder mejorarla.

\section{Participantes}

La población objeto de estudio está compuesta por 108 estudiantes, matriculados durante el curso académico 2012/13 en el Máster Universitario en profesorado de Educación Secundaria Obligatoria y Bachillerato, Formación Profesional y Enseñanza de Idiomas de la Universidad de Jaén. Para la selección de los sujetos se ha utilizado un muestreo probabilístico aleatorio simple, de forma que se han adscrito a la muestra todos aquellos sujetos que al azar respondieron a los cuestionarios elaborados para tal fin $(n=99)$. Para el cálculo de la muestra participante se utilizó la fórmula para poblaciones con menos de 100.000 sujetos con un nivel de confianza del $95 \%$ y un error de estimación máximo de un $4 \%$. En la tabla $n^{\circ} 1$ se expone, de manera resumida, la distribución de la muestra según variables sociodemográficas estudiadas:

\begin{tabular}{|c|c|c|c|}
\hline & & $\mathbf{n}$ & $\%$ \\
\hline \multirow{2}{*}{ Género } & Hombre & 39 & 39.4 \\
\hline & Mujer & 60 & 60.6 \\
\hline \multirow{3}{*}{ Edad } & Menos de 25 años & 63 & 63.6 \\
\hline & Entre 26 y 30 años & 16 & 16.2 \\
\hline & Más de 31 años & 20 & 20.2 \\
\hline \multirow{5}{*}{$\begin{array}{l}\text { Titulación de } \\
\text { acceso }\end{array}$} & Ciencias Experimentales & 10 & 10.1 \\
\hline & Ciencias de la Salud & 4 & 4.0 \\
\hline & Enseñanzas técnicas & 18 & 18.2 \\
\hline & Ciencias Sociales y Jurídicas & 7 & 7.1 \\
\hline & Humanidades & 60 & 60.6 \\
\hline \multirow{10}{*}{ Especialidad } & Biología y Geología & 11 & 11.1 \\
\hline & Dibujo, imagen y artes plásticas & 6 & 6.1 \\
\hline & Economía, empresa y comercio & 5 & 5.1 \\
\hline & Formación y orientación laboral & 3 & 3.0 \\
\hline & Ciencias Sociales & 6 & 6.1 \\
\hline & Tecnología y procesos industriales & 11 & 11.1 \\
\hline & Lengua extranjera & 34 & 34.3 \\
\hline & Lengua y literatura & 9 & 9.1 \\
\hline & Matemáticas e Informática & 3 & 3.0 \\
\hline & Orientación educativa & 11 & 11.1 \\
\hline \multirow{3}{*}{$\begin{array}{l}\text { Motivo de } \\
\text { acceso al } \\
\text { Máster }\end{array}$} & Vocación & 45 & 34.6 \\
\hline & Prestigio & 2 & 1.5 \\
\hline & Posibilidad de acceder a un trabajo estable & 63 & 48.5 \\
\hline
\end{tabular}




\begin{tabular}{llll}
\hline $\begin{array}{l}\text { Por no tener una mejor opción en la salida } \\
\text { de mi titulación }\end{array}$ & 4 & 3.1 \\
\hline $\begin{array}{l}\text { Influencia de algún familiar vinculado a la } \\
\text { docencia }\end{array}$ & 8 & 6.2 \\
\hline $\begin{array}{l}\text { Influencia de algún profesor que me ha } \\
\text { marcado en mi formación }\end{array}$ & 6 & 4.6 \\
\hline Diversificar la docencia & 2 & 1.5 \\
\hline
\end{tabular}

Tabla 1. Caracterización de la muestra.

\section{Instrumento}

Para la recogida de datos, se ha utilizado un cuestionario elaborado ad hoc. Dicho instrumento, compuesto de 43 ítems, consiste en una escala tipo Likert cuyas opciones de respuesta oscilan entre 1 y 4 (siendo $1=$ plenamente de acuerdo, $2=$ de acuerdo; $3=$ en desacuerdo y $4=$ totalmente en desacuerdo).

Construida la escala, se procedió a la validación por parte de una docena de expertos universitarios en Pedagogía de la Universidad de Jaén y Granada a los que se les pidió que valoraran y validaran la pertinencia de cada uno de los ítems del cuestionario, estableciendo las consideraciones oportunas para mejorarlo en cuanto a su contenido, ambigüedad, redacción y otros aspectos. Una vez estas sugerencias fueron incorporadas al instrumento, se procedió a la aplicación de una prueba piloto a 90 estudiantes universitarios que se encontraban cursando dicho Máster en otras universidades andaluzas. Esta prueba tenía como intención conocer el grado de comprensión de los distintos ítems por parte del alumnado y analizar la validez de constructo (análisis factorial mediante la extracción de componentes principales con rotación Varimax). Así, el índice de adecuación muestral KMO alcanza un valor de .800 y la prueba de esfericidad de Bartlett es de 3277.395 ( $\mathrm{p}=.000$ ). Estos datos hacen que se rechace la hipótesis nula de que la matriz de correlación inter-ítems es identidad y se consideran que las respuestas están sustancialmente relacionadas. El análisis de los componentes principales revela, tras la rotación Varimax, la convergencia en 5 factores que explican el $60.80 \%$ de la varianza; los ítems muestran valores apropiados, situándose entre .564 y .853 .

En cuanto a la fiabilidad del instrumento, se ha utilizado el método alpha de Cronbach obteniéndose un valor de .915. Por ello, se puede deducir que el cuestionario elaborado para tal investigación tiene una fiabilidad muy alta, ya que el coeficiente está próximo a 1, considerada la correlación perfecta. Para asegurar aún más la fiabilidad del instrumento, se aplicó el método de las dos mitades, obteniéndose puntuaciones muy apropiadas, pues en la primera parte se obtuvo un valor de .842 y para la segunda el valor obtenido fue de .906 .

\section{Procedimiento}

El plan de estudios del Máster Universitario en profesorado de Educación Secundaria Obligatoria y Bachillerato, Formación Profesional y Enseñanza Idiomas de 
la Universidad de Jaén, consta de un módulo genérico en el que se incluye la asignatura denominada "Procesos y contextos educativos"; es en esta materia dónde tiene cabida el estudio de contenidos propios del ámbito de la Educación Especial y la atención a la diversidad en la formación de los docentes de Educación Secundaria.

Por ello, y para la aplicación del cuestionario a la muestra, se tuvo en cuenta el que los estudiantes hubiesen cursado ya esta materia obligatoria para todas las especialidades durante el primer cuatrimestre del curso 2012/13. Asimismo, se contó con el visto bueno de los coordinadores de este Máster, los cuáles nos facilitaron el acceso a la muestra. Cada uno de los estudiantes recibió las explicaciones necesarias para cumplimentar el cuestionario de manera adecuada.

\section{Análisis de datos}

Para el tratamiento estadístico de los datos se ha utilizado el programa SPSS (versión 19 para Windows) al considerarlo un recurso idóneo para nuestro trabajo. De este modo, se ha llevado a cabo un análisis descriptivo de los resultados obtenidos en los distintos ítems del cuestionario, agrupados en los factores de los que consta el mismo. A continuación, y con la intención de conocer el grado de relación entre estos y las variables más directamente vinculadas con la formación que ofrece el Máster en aspectos referidos al ámbito de la Educación Especial se ha utilizado el coeficiente de correlación de Pearson y análisis de regresión múltiple lineal para conocer si existe influencia entre las variables predictoras y criterio.

\section{Resultados}

Con la intención de analizar las valoraciones de los estudiantes del Máster sobre atención a la diversidad, se han examinado las puntuaciones de las medias y desviaciones típicas obtenidas en cada uno de los ítems de los diferentes factores que componen el cuestionario.

\section{Elementos condicionantes del proceso de atención a la diversidad en el aula}

Las puntuaciones obtenidas por los ítems que forman parte de este factor, se sitúan por debajo de la media, es decir, todas poseen un valor menor a 2 puntos, lo cual induce a considerar que el alumnado encuestado se muestra muy favorable con las proposiciones planteadas en el cuestionario (tabla $\mathrm{n}^{\circ} 2$ ). Así pues, los estudiantes del Máster se muestran muy favorables a la hora de considerar la atención a la diversidad como un deber del centro educativo, capaz de enriquecer a toda la comunidad educativa. De igual modo, consideran que la atención a la diversidad requiere de un trabajo colaborativo entre todos los profesionales del centro, de la coordinación entre todos ellos y de la motivación e interés profesional del docente, poniendo de manifiesto cómo la atención al alumnado con necesidades educativas es responsabilidad de todo el profesorado que lo atiende. En su futura práctica docente, los encuestados se muestran muy convencidos de que la atención a la diversidad debe ocupar un lugar destacado, 
siendo una de sus mayores preocupaciones lograr la plena inclusión del alumnado en el aula. Los alumnos que cursan el Máster de Secundaria consideran que la actitud de la familia supone un aspecto muy influyente en la calidad de la educación del alumnado con necesidades específicas de apoyo educativo. De igual modo, consideran relevante la labor del Departamento de Orientación o el apoyo de los órganos de dirección del centro en la respuesta a las necesidades educativas del alumnado con discapacidad. También los encuestados se muestran muy de acuerdo en definir la formación inicial del docente, la disponibilidad de recursos humanos y materiales o una adecuada ratio profesor-alumno como elementos fundamentales para llevar a cabo un proceso de atención a la diversidad de calidad. Se destaca, además, el acuerdo de los encuestados en considerar que la atención a la diversidad pueda llevarse a cabo en todas y cada una de las áreas que componen el currículum de Educación Secundaria. Finalmente, se muestra el alto acuerdo de los estudiantes a la hora de suponer cómo debería concederse más importancia a la atención a la diversidad en los planes de estudio del Máster que están cursando, considerando muy eficaz la combinación de modalidades de escolarización para el alumnado con necesidades educativas entre el aula de apoyo y el aula ordinaria.

\begin{tabular}{|c|c|c|}
\hline $\begin{array}{l}\text { Elementos condicionantes del proceso de atención a la diversidad en el } \\
\text { aula }\end{array}$ & M. & D.T. \\
\hline Es un deber del centro educativo atender a todo el alumnado & 1,46 & 1,003 \\
\hline $\begin{array}{l}\text { Para una mejor atención a la diversidad se requiere trabajar de manera } \\
\text { colaborativa entre los profesionales del centro educativo }\end{array}$ & 1,56 & 0,883 \\
\hline $\begin{array}{l}\text { Un proceso de atención a la diversidad de calidad requiere de una } \\
\text { coordinación entre el profesorado }\end{array}$ & 1,58 & 0,938 \\
\hline $\begin{array}{l}\text { Un proceso de atención a la diversidad de calidad requiere de motivación e } \\
\text { interés profesional del docente }\end{array}$ & 1,61 & 0,879 \\
\hline $\begin{array}{l}\text { La actitud de la familia influye en la calidad de la educación del alumnado con } \\
\text { n.e.a.e. }\end{array}$ & 1,61 & 0,924 \\
\hline La atención al alumnado con n.e.a.e. es responsabilidad de todo el profesorado & 1,63 & 0,864 \\
\hline $\begin{array}{l}\text { La atención a la diversidad debe ocupar un papel importante en mi futura } \\
\text { práctica docente }\end{array}$ & 1,64 & 0,942 \\
\hline $\begin{array}{l}\text { Considero fundamental la labor del Departamento de Orientación en la } \\
\text { respuesta a las necesidades educativas del alumnado con discapacidad }\end{array}$ & 1,68 & 0 \\
\hline $\begin{array}{l}\text { Como docente, mi preocupación es que los alumnos logren su inclusión en el } \\
\text { aula }\end{array}$ & 1,70 & 0,874 \\
\hline $\begin{array}{l}\text { En la atención a la diversidad, el apoyo de los órganos de dirección del centro } \\
\text { es prioritario }\end{array}$ & 1,71 & 0,773 \\
\hline $\begin{array}{l}\text { Un proceso de atención a la diversidad de calidad requiere: formación inicial } \\
\text { sobre atención a la diversidad }\end{array}$ & 1,73 & 0,831 \\
\hline $\begin{array}{l}\text { Un proceso de atención a la diversidad de calidad requiere de recursos } \\
\text { suficientes y apropiados }\end{array}$ & 1,73 & 0,843 \\
\hline La atención a la diversidad en el aula enriquece a toda la comunidad educativa & 1,74 & 0,954 \\
\hline
\end{tabular}




\begin{tabular}{lcc}
\hline $\begin{array}{l}\text { La atención a la diversidad puede llevarse a cabo en todas y cada una de las } \\
\text { áreas que componen el currículum de Secundaria }\end{array}$ & 1,76 & 0,784 \\
\hline $\begin{array}{l}\text { Un proceso de atención a la diversidad de calidad requiere de una adecuada } \\
\text { ratio profesor-alumno }\end{array}$ & 1,82 & 0,952 \\
\hline $\begin{array}{l}\text { Se le debería conceder una mayor importancia a la atención a la diversidad en } \\
\text { el Plan de Estudios que estoy cursando }\end{array}$ & 1,96 & 0,794 \\
\hline $\begin{array}{l}\text { Lo más eficaz para atender la diversidad es combinar la escolarización del } \\
\text { alumnado con n.e.a.e. entre el aula ordinaria y el aula de apoyo }\end{array}$ & 1,97 & 0,839 \\
\hline
\end{tabular}

Tabla 2. Análisis descriptivo Factor 1.

\section{Respuesta curricular y organizativa a la diversidad en el aula}

La tabla n 3 muestra cómo, para este factor, las puntuaciones revelan la satisfacción de los encuestados respecto a las proposiciones planteadas en la escala. Así pues, el alumnado que cursa el Máster de Secundaria manifiesta haber recibido una formación adecuada sobre atención a la diversidad respecto al agrupamiento de los alumnos, selección y adaptación de objetivos, contenidos y competencias básicas, medidas y programas de atención a la diversidad puestos en marcha desde el sistema educativo, organización del espacio, selección, diseño y práctica de actividades y tareas académicas, estrategias metodológicas, evaluación del proceso de enseñanza y aprendizaje y organización del tiempo.

\begin{tabular}{lcc}
\hline \multicolumn{1}{c}{ Respuesta curricular y organizativa a la diversidad en el aula } & M. & D.T. \\
\hline Agrupamiento de alumnos & 2,33 & 0,756 \\
\hline Selección y adaptación de objetivos, competencias y contenidos & 2,40 & 0,699 \\
\hline Medidas y programas de atención a la diversidad & 2,43 & 0,758 \\
\hline Organización del espacio & 2,44 & 0,703 \\
\hline Selección, diseño y práctica de actividades y tareas & 2,45 & 0,746 \\
\hline Estrategias metodológicas & 2,46 & 0,760 \\
\hline Evaluación del proceso de enseñanza y aprendizaje & 2,46 & 0,704 \\
\hline Organización del tiempo & 2,48 & 0,705 \\
\hline
\end{tabular}

Tabla 3. Análisis descriptivo Factor 2.

\section{Capacitación docente hacia la diversidad adquirida en el Máster}

La tabla $\mathrm{n}^{\circ} 4$ muestra cómo para este factor las puntuaciones obtenidas se sitúan alrededor de la media (2 puntos), lo cual supone el acuerdo de los encuestados respecto a las proposiciones planteadas en el cuestionario. Así, los estudiantes de esta titulación de postgrado expresan su acuerdo a la hora de considerar cómo los estudios de Máster realizados le han servido para aumentar su interés hacia la formación de este ámbito, desarrollar una mayor sensibilización hacia la diversidad y para afianzar su elección profesional hacia la docencia. De igual modo, sostienen cómo, tras cursar el módulo genérico del Máster, y más concretamente, la asignatura "Procesos y contextos educativos" han obtenido un conocimiento adecuado sobre la identificación de las 
necesidades educativas en el alumnado, recursos y servicios prestados desde el sistema educativo, características de los alumnos con necesidades educativas, aspectos básicos del campo de la Educación Especial y legislación sobre el ámbito de la atención a la diversidad. No obstante, consideran que la formación sobre Educación Especial recibida no ha sido suficiente.

\begin{tabular}{lcc}
\hline \multicolumn{1}{c}{ Capacitación docente hacia la diversidad adquirida en el Máster } & M. & D.T. \\
\hline $\begin{array}{l}\text { Este Máster me ha servido para aumentar mi interés hacia una mayor formación en } \\
\text { el ámbito de la atención a la diversidad }\end{array}$ & 2,19 & 0,888 \\
\hline $\begin{array}{l}\text { Este Máster me ha servido para tener una mayor sensibilización hacia la atención a } \\
\text { la diversidad }\end{array}$ & 2,19 & 0,900 \\
\hline Este Máster me ha servido para afianzar mi elección profesional hacia la docencia & 2,21 & 0,860 \\
\hline $\begin{array}{l}\text { Tras cursar el módulo genérico del Máster, poseo un conocimiento adecuado sobre } \\
\text { la identificación de necesidades educativas en el alumnado }\end{array}$ & 2,29 & 0,759 \\
\hline $\begin{array}{l}\text { Tras cursar el módulo genérico del Máster, tengo un conocimiento adecuado sobre } \\
\text { los recursos y servicios prestados desde el sistema educativo }\end{array}$ & 2,33 & 0,670 \\
\hline $\begin{array}{l}\text { Tras cursar el módulo genérico del Máster, poseo un conocimiento adecuado sobre } \\
\text { las características de los alumnos con necesidades educativas }\end{array}$ & 2,34 & 0,717 \\
\hline $\begin{array}{l}\text { Tras cursar el módulo genérico del Máster, tengo un conocimiento adecuado sobre } \\
\text { los aspectos básicos del campo de la Educación Especial }\end{array}$ & 2,36 & 0,735 \\
\hline $\begin{array}{l}\text { Tras cursar el módulo genérico del Máster, poseo un conocimiento adecuado sobre } \\
\text { legislación relativa al ámbito de la Educación Especial }\end{array}$ & 2,43 & 0,688 \\
\hline $\begin{array}{l}\text { En el Máster que estoy cursando he adquirido suficientes conocimientos sobre } \\
\text { Educación Especial }\end{array}$ & 2,58 & 0,846 \\
\hline
\end{tabular}

Tabla 4. Análisis descriptivo Factor 3.

\section{Práctica docente formativa en la atención a la diversidad}

La tabla $n^{\circ} 5$ recoge los resultados obtenidos para los ítems de este factor, situándose las puntuaciones obtenidas alrededor de la media (mayor de 2 puntos), lo que indica el acuerdo de los encuestados respecto a las afirmaciones planteadas en la escala. De este modo, el alumnado encuestado se muestra favorable en considerar que el proceso de atención a la diversidad requiere de experiencia previa por parte del docente con alumnos con necesidades específicas de apoyo educativo. De igual modo, y tras cursar los módulos genéricos del Máster, manifiestan que se encuentran suficientemente capacitados como para afrontar el reto de la diversidad en el aula, considerando que el desarrollo de actividades y ejemplos prácticos ha mejorado sus conocimientos sobre este campo de estudio sintiéndose capaces de dar respuesta a las situaciones que se les puedan plantear con alumnado con necesidades específicas de apoyo educativo. No obstante, se encuentran menos convencidos de que el sistema educativo ofrece los servicios y recursos adecuados para atender las necesidades educativas del alumnado escolarizado. 


\begin{tabular}{lcc}
\hline \multicolumn{1}{c}{ Práctica docente formativa en la atención a la diversidad } & M. & D.T. \\
\hline $\begin{array}{l}\text { Un proceso de atención a la diversidad de calidad requiere de } \\
\text { experiencia previa con alumnos con necesidades educativas }\end{array}$ & 2,01 & 0,827 \\
\hline $\begin{array}{l}\text { Me considero suficientemente capacitado como para afrontar el reto } \\
\text { de la diversidad en mi aula }\end{array}$ & 2,43 & 0,797 \\
\hline $\begin{array}{l}\text { Las actividades o ejemplos prácticos desarrollados en las clases han } \\
\text { mejorado mis conocimientos sobre atención a la diversidad }\end{array}$ & 2,46 & 0,825 \\
\hline $\begin{array}{l}\text { Los conocimientos adquiridos sobre atención a la diversidad me } \\
\text { darán respuesta a situaciones que se puedan plantear con alumnos } \\
\text { con n.e.a.e. }\end{array}$ & 2,51 & 0,761 \\
\hline $\begin{array}{l}\text { Desde el sistema educativo, se ofrecen los servicios y recursos } \\
\text { adecuados para atender las necesidades de los alumnos }\end{array}$ & 2,55 & 0,746 \\
\hline
\end{tabular}

Tabla 5. Análisis descriptivo Factor 4.

\section{Percepción docente hacia el alumnado con necesidades específicas de apoyo educativo}

La tabla $n^{\circ} 6$ refleja cómo las puntuaciones obtenidas se sitúan alrededor de la media (mayor de 2 puntos), lo cual revela el acuerdo de los encuestados respecto a las proposiciones planteadas en el cuestionario. Así pues, los estudiantes del Máster de Educación Secundaria consideran que los alumnos con necesidades específicas de apoyo educativo precisan de numerosas modificaciones en el currículum, sosteniendo que su atención supone un trabajo añadido para el profesor. No obstante, se muestran menos favorables en definir como una utopía el hecho de conseguir una adecuada atención a la diversidad en Educación Secundaria, así como en considerar los centros de educación especial como la mejor modalidad de escolarización para el alumnado con necesidades específicas.

\begin{tabular}{lcc}
\hline $\begin{array}{c}\text { Percepción docente hacia el alumnado con necesidades específicas de } \\
\text { apoyo educativo }\end{array}$ & M. & D.T. \\
\hline $\begin{array}{l}\text { Los alumnos con n.e.a.e. precisan de numerosas modificaciones en el } \\
\text { currículum }\end{array}$ & 2,20 & 0,714 \\
\hline $\begin{array}{l}\text { Trabajar con alumnos n.e.a.e es un trabajo añadido para el profesor tutor } \\
\text { en el aula }\end{array}$ & 2,32 & 0,867 \\
\hline $\begin{array}{l}\text { Conseguir una adecuada atención a la diversidad en E.S.O. resulta algo } \\
\text { utópico }\end{array}$ & 2,64 & 0,863 \\
\hline $\begin{array}{l}\text { Los alumnos con n.e.a.e. estarían mejor atendidos en centros de Educación } \\
\text { Especial }\end{array}$ & 2,65 & 0,918 \\
\hline
\end{tabular}

Tabla 6. Análisis descriptivo Factor 5.

A continuación, se analiza la relación entre los factores que agrupan los ítems del cuestionario mediante la correlación $r$ de Pearson (tabla $n^{\circ} 7$ ). Los resultados obtenidos demuestran cómo existe correspondencia entre la respuesta organizativa y curricular a la diversidad en el aula y la capacitación docente sobre procesos de atención a la 
diversidad adquirida por los estudiantes en el Máster (.605), al igual que con la práctica docente formativa en la atención a la diversidad (.502). También se comprueba reciprocidad entre este último factor y la capacitación docente sobre procesos de atención a la diversidad adquirida en el Máster (.508). No obstante, la revisión de la tabla pone de manifiesto cómo los elementos condicionantes de la atención a la diversidad (la variable que más ítems aglutina) y la percepción docente hacia el alumnado con necesidades específicas de apoyo educativo, no guardan relación con el resto.

\begin{tabular}{llcccc}
\hline & \multicolumn{3}{c}{ r } \\
\cline { 2 - 6 } & F1 & F2 & F3 & F4 & F5 \\
\hline $\begin{array}{l}\text { F1.Elementos condicionantes del proceso de atención a } \\
\text { la diversidad en el aula }\end{array}$ & & .127 & .102 & .196 & -.029 \\
\hline $\begin{array}{l}\text { F2.Respuesta curricular y organizativa a la diversidad } \\
\text { en el aula }\end{array}$ & .125 & & $.605^{* *}$ & $.502^{* *}$ &.-054 \\
\hline $\begin{array}{l}\text { F3. Capacitación docente sobre procesos de atención a } \\
\text { la diversidad adquiridas en el Máster }\end{array}$ & .102 & $.605^{* *}$ & & $.508^{* *}$ &.-026 \\
\hline $\begin{array}{l}\text { F4.Práctica docente formativa en la atención a la } \\
\text { diversidad }\end{array}$ & .196 & $.502^{* *}$ & $.508^{* *}$ & & -.074 \\
\hline $\begin{array}{l}\text { F5.Percepción docente hacia el alumnado con } \\
\text { necesidades específicas de apoyo educativo }\end{array}$ & -.029 & -.054 & -.026 & -.074 & \\
\hline
\end{tabular}

Tabla 7. Correlaciones entre factores.

En un segundo lugar, se analizan las correlaciones entre las variables más directamente vinculadas con la formación que ofrece el Máster en relación con la atención a la diversidad y la Educación Especial, siendo éstas: la atención a la diversidad debe ocupar un papel importante en mi futura práctica docente (v11), en el Máster que estoy cursando he adquirido suficientes conocimientos sobre Educación Especial (v26), este Máster me ha servido para afianzar mi elección profesional hacia la docencia (v34), este Máster me ha servido para tener una mayor sensibilización hacia la atención a la diversidad (v35) y este Máster me ha servido para aumentar mi interés hacia una mayor formación en el ámbito de la atención a la diversidad (v36) (tabla $\mathrm{n}^{\circ} 8$ ). Los resultados obtenidos señalan cómo las mayores correlaciones se corresponden con el hecho de que el Máster sirva para aumentar la sensibilidad hacia la atención a la diversidad y el interés hacia la formación en esta temática (.732); además de la utilidad de esta formación para aumentar el interés en la preparación para hacer frente a la atención a la diversidad y afianzar la elección profesional hacia la docencia (.694). 


\begin{tabular}{llcccc}
\hline & & & r & \\
& V11 & V26 & V34 & V35 & V36 \\
\hline $\begin{array}{l}\text { V11. La atención a la diversidad debe ocupar un papel } \\
\text { importante en mi futura práctica docente }\end{array}$ & & & & $.218^{*}$ \\
\hline $\begin{array}{l}\text { V26. En el Máster que estoy cursando he adquirido } \\
\text { suficientes conocimientos sobre Educación Especial }\end{array}$ & & $.349^{* *}$ & $.376^{* *}$ & $.381^{* *}$ \\
\hline $\begin{array}{l}\text { V34. Este Máster me ha servido para afianzar mi } \\
\text { elección profesional hacia la docencia }\end{array}$ & $.349^{* *}$ & & .593 & $.694^{* *}$ \\
\hline $\begin{array}{l}\text { V35. Este Máster me ha servido para tener una mayor } \\
\text { sensibilización hacia la atención a la diversidad }\end{array}$ & $.376^{* *}$ & $.593^{* *}$ & & $.732^{* *}$ \\
\hline $\begin{array}{l}\text { V36. Este Máster me ha servido para aumentar mi } \\
\text { interés hacia una mayor formación en el ámbito de la } \\
\text { atención a la diversidad }\end{array}$ & & & & & \\
\hline
\end{tabular}

Tabla 8. Correlaciones entre ítems más significativos.

Un paso más en el análisis nos lleva a considerar la fuerza global en la asociación entre las variables vinculadas directamente con los aspectos formativos y los factores que agrupan los ítems de la escala. Con tal fin se lleva a cabo una regresión múltiple lineal para comprobar el valor predictor de las siguientes variables independientes: edad, titulación de acceso, especialidad y aquellos ítems del cuestionario que más directamente están relacionados con aspectos formativos.

El factor 1 "Elementos condicionantes del proceso de atención a la diversidad en el aula" ha obtenido un coeficiente de determinación alto, al igual que una relación significativa entre las variables analizadas. En la Tabla $n^{\circ} 9$ se aprecia cómo la variable que tiene más fuerza en la predicción de los elementos que condicionan la atención a la diversidad es el hecho de considere a ésta como importante en la futura práctica docente $(\mathrm{t}=13.40, \mathrm{p} \leq .000)$.

\begin{tabular}{|c|c|c|c|c|}
\hline & \multicolumn{2}{|c|}{ Coef. No estand. } & \multirow{2}{*}{$\begin{array}{l}\text { Coef. Tipif. } \\
\text { Beta (stand) }\end{array}$} & \multirow{2}{*}{$\mathbf{t}$} \\
\hline & Beta & E.T. & & \\
\hline (Constante) & 15.17 & 2.83 & & $5.36^{*}$ \\
\hline Edad & -1.35 & .916 & -.094 & $-1,47$ \\
\hline Titulación de acceso & -.25 & .087 & -.018 & -.291 \\
\hline Especialidad & -.13 & .17 & -.045 & -.744 \\
\hline V11 & 9.78 & .730 & .794 & $13.40 * *$ \\
\hline V26 & -1.26 & .913 & -.092 & -1.38 \\
\hline V34 & .173 & 1.10 & .013 & .156 \\
\hline V35 & -.29 & 1.14 & -.022 & -.253 \\
\hline $\mathrm{V} 36$ & 1.44 & 1.29 & .110 & 1.11 \\
\hline
\end{tabular}

Tabla 9. Regresión múltiple lineal para la variable Factor 1. 
En el factor 2 se constata un modelo de regresión con una menor fuerza global con las variables predictoras. Estos datos señalan un coeficiente de determinación más bajo que en el factor anterior; así pues, la variable 26, "En el Máster que estoy cursando he adquirido conocimientos suficientes de Educación Especial" actúa como predictora del factor "Respuesta curricular y organizativa a la diversidad en el aula" ( $\mathrm{t}=3.86$, $\mathrm{p} \leq .000)\left(\right.$ tabla $\left.^{\circ} 10\right)$.

\begin{tabular}{|c|c|c|c|c|}
\hline & \multicolumn{2}{|c|}{ Coef. No estand. } & \multirow{2}{*}{$\begin{array}{l}\text { Coef. Tipif. } \\
\text { Beta (stand) } \\
\end{array}$} & \multirow{2}{*}{$\mathbf{t}$} \\
\hline & Beta & E.T. & & \\
\hline (Constante) & 8.471 & 1.63 & & $5.197 * *$ \\
\hline Edad & .968 & .527 & .170 & 1.835 \\
\hline Titulación de acceso & .038 & .050 & .068 & .760 \\
\hline Especialidad & .034 & .102 & .030 & .336 \\
\hline V11 & .669 & .420 & .136 & 1.593 \\
\hline V26 & 2.032 & .526 & .372 & $3.866^{* *}$ \\
\hline V34 & .865 & .638 & .161 & 1.356 \\
\hline $\mathrm{V} 35$ & .183 & .660 & .036 & .277 \\
\hline V36 & .537 & .744 & .103 & .721 \\
\hline
\end{tabular}

Tabla 10. Regresión múltiple lineal para la variable Factor 2.

El factor 3 hace referencia a la "Capacitación docente sobre procesos de atención a la diversidad adquiridas en el Máster”; en este caso la fuerza de la asociación es muy alta, tal y como se aprecia en el modelo de regresión. Estos datos muestran un coeficiente de determinación alto, al igual que una relación significativa entre las variables analizadas. Tal y como se aprecia en la tabla $n^{\circ} 11$, las variables que mejor predicen este factor son las que hacen referencia a la adquisición de conocimientos (v26) $(\mathrm{t}=7.074, \mathrm{p}<.000)$, el hecho de que el Máster haya servido para afianzar la elección profesional hacia la docencia $(\mathrm{v} 34)(\mathrm{t}=3.658, \mathrm{p} \leq .000)$, la mejora de la sensibilización hacia la atención a la diversidad ( $\mathrm{v} 35)(\mathrm{t}=4.529, \mathrm{p} \leq .000)$ y el aumento del interés hacia una mayor formación en el ámbito de la atención a la diversidad (v36) $(\mathrm{t}=3.429, \mathrm{p} \leq .001)$. En definitiva, la predicción de este factor, que aglutina variables vinculadas con la formación recibida, ha sido muy alta por parte de las variables que hacen referencia a esta cuestión, tal y como muestra el gráfico de regresión. 


\begin{tabular}{|c|c|c|c|c|}
\hline & \multicolumn{2}{|c|}{ Coef. No estand. } & \multirow{2}{*}{$\begin{array}{l}\text { Coef. Tipif. } \\
\text { Beta (stand) } \\
\end{array}$} & \multirow{2}{*}{$\mathbf{t}$} \\
\hline & Beta & E.T. & & \\
\hline (Constante) & 5.665 & .909 & & $6.230 * *$ \\
\hline Edad & .352 & .294 & .057 & 1.197 \\
\hline Titulación de acceso & .032 & .028 & .053 & 1.143 \\
\hline Especialidad & -.045 & .057 & -.036 & -.788 \\
\hline V11 & .043 & .234 & .008 & .186 \\
\hline $\mathrm{V} 26$ & 2.074 & .293 & .353 & $7.074 * *$ \\
\hline V34 & 1.301 & .356 & .225 & $3.658 * *$ \\
\hline V35 & 1.667 & .368 & .302 & $4.529 * *$ \\
\hline V36 & 1.423 & .415 & .254 & $3.429 *$ \\
\hline
\end{tabular}

Tabla 11. Regresión múltiple lineal para la variable Factor 3.

En el factor 4 se comprueba un modelo de regresión con una menor fuerza global con las variables predictoras. Estos datos constatan un coeficiente de determinación más bajo que en el factor anterior. Las variables en las que se confiere importancia a la atención a la diversidad en la práctica docente (v11) y la satisfacción con los conocimientos adquiridos sobre Educación Especial (v26) actúan como predictoras del "Práctica docente formativa en la atención a la diversidad" $(\mathrm{t}=3.279, \mathrm{p} \leq .001)$ y $(\mathrm{t}=5.309, \mathrm{p} \leq .000)\left(\operatorname{tabla~}^{\circ} 12\right)$.

\begin{tabular}{lcccc}
\hline & \multicolumn{2}{c}{ Coef. No estand. } & Coef. Tipif. & \multirow{2}{*}{ t } \\
\cline { 1 - 4 } & Beta & E.T. & Beta (stand) & \\
\hline (Constante) & 6.497 & .798 & & $8.141^{* *}$ \\
\hline Edad & .252 & .258 & .089 & .974 \\
\hline Titulación de acceso & .004 & .025 & .013 & .149 \\
\hline Especialidad & .012 & .025 & .020 & .233 \\
\hline V11 & .674 & .206 & .277 & $3.279^{*}$ \\
\hline V26 & 1.366 & .257 & .504 & $5.309 * *$ \\
\hline V34 & .217 & .312 & .081 & .696 \\
\hline V35 & -.310 & .323 & -.122 & -.960 \\
\hline V36 & .354 & .364 & .137 & .971 \\
\cline { 1 - 4 }
\end{tabular}

Tabla 12. Regresión múltiple lineal para la variable Factor 4.

El último de los factores es el que menos fuerza global presenta en la asociación con las variables predictoras. Esta $\mathrm{F}$ de Snedecor tan baja indica claramente que no existe relación significativa entre las variables predictoras y la criterio para el factor 5 denominado "Percepción docente hacia los alumnos con necesidades específicas de apoyo educativo" (tabla 13). 


\begin{tabular}{|c|c|c|c|c|}
\hline & \multicolumn{2}{|c|}{ Coef. No estand. } & \multirow{2}{*}{$\begin{array}{l}\text { Coef. Tipif. } \\
\text { Beta (stand) } \\
\end{array}$} & \multirow{2}{*}{$\mathbf{t}$} \\
\hline & Beta & E.T. & & \\
\hline (Constante) & 10.822 & .897 & & $12.059 * *$ \\
\hline Edad & -.136 & .290 & -.054 & -.469 \\
\hline Titulación de acceso & .018 & .028 & .075 & .662 \\
\hline Especialidad & -.016 & .056 & -.032 & -.293 \\
\hline V11 & -.215 & .231 & -.100 & -932 \\
\hline $\mathrm{V} 26$ & .080 & .289 & .034 & .278 \\
\hline V34 & -.411 & .351 & -.175 & -1.170 \\
\hline V35 & -.018 & .363 & -.008 & -.048 \\
\hline V36 & -038 & .410 & .016 & .092 \\
\hline
\end{tabular}

Tabla 13. Regresión múltiple lineal para la variable Factor 5.

\section{Conclusiones}

Con este trabajo se han pretendido analizar las percepciones del alumnado que cursa el Máster Universitario en Profesorado de Educación Secundaria Obligatoria, Formación Profesional y Enseñanza de Idiomas acerca de la formación inicial recibida sobre atención a la diversidad. Así pues, los estudiantes participantes en la investigación se han mostrado favorables hacia la determinación de una serie de aspectos condicionantes del proceso de atención a la diversidad en el aula tales como: trabajo colaborativo entre profesionales, motivación docente, actitud de la familia, apoyo de los órganos de dirección, adecuada ratio-profesor alumno, etc. De este modo, se evidencia la buena predisposición de los futuros docentes de Educación Secundaria hacia el desarrollo de un proceso de atención a la diversidad de calidad, capaz de lograr la plena inclusión del alumnado con necesidades específicas de apoyo educativo en el aula. El Máster es concebido, desde este punto de vista, como una nueva oportunidad formativa para los profesores de Educación Secundaria Obligatoria (González Sanmmamed, 2009) que les puede llevar a la adquisición de unos conocimientos didácticos y psicopedagógicos más profundos que les capacite para su futuro ejercicio profesional en el aula (Buendía et al., 2011). No obstante, autores como Sánchez (2007) establecen cómo el docente, antes de su práctica diaria en el aula, ya parte de prejuicios, creencias e ideas que dificultan dicho proceso.

No obstante, los encuestados consideran que la formación recibida sobre aspectos curriculares y organizativos hacia la diversidad es la adecuada en cuestiones vinculadas al agrupamiento del alumnado, selección y adaptación de objetivos, contenidos y competencias básicas, medidas y programas de atención a la diversidad ofrecidos desde el sistema educativo español, organización espacio-temporal, estrategias metodológicas y diseño de actividades y su posterior evaluación. Así pues, tal y como establece Reoyo, Carbonero, Freitas y Valdivieso (2012) resulta de vital importancia el que el docente esté formado tanto en aspectos curriculares como afectivos y habilidades relacionales. Sin embargo, estos datos entran en contradicción con los aportados por Boix (2008) quién demuestra el desconocimiento de los futuros docentes de Educación Secundaria acerca del concepto de atención a la diversidad, estrategias y recursos de 
integración e inclusión, competencias metodológicas y entornos de comunidad de aprendizaje.

La intencionalidad del Máster, por tanto, se vincula con un aumento de la formación y sensibilización docente hacia la atención a la diversidad, capaz de afianzar la elección del estudiante hacia el ámbito de la docencia. Esta finalidad, por tanto, ha de dirigirse hacia una mejor preparación y desarrollo profesional del docente en el ámbito de la atención a la diversidad, capaz de superar los retos diarios que se le plantean en su actividad en el aula (Buendía et al., 2011). Varios estudios señalan el factor docente como elemento clave de la transformación educativa, actor principal de reforma de los modelos de enseñanza (Aguerrondo, 2004; Fullan, 2002; Vaillant, 2009). Por ello, resulta necesario, entre otras acciones, implementar políticas que posibiliten su desarrollo profesional y la mejora de sus condiciones laborales así como revisar los sistemas de formación y las claves de la profesión según los nuevos escenarios sociales y culturales (Vezub, 2007).

Por ello, los estudiantes sostienen cómo el hecho de haber cursado el módulo genérico vinculado al ámbito de la Educación Especial, les ha reportado conocimientos acerca de las necesidades educativas del alumnado, recursos y servicios prestados, legislación, así como aspectos básicos de este campo de estudio. Sin duda, unas percepciones bastante positivas que contradicen los resultados de distintas investigaciones en las que se concluye la falta de formación inicial demandada por los futuros docentes de Educación Secundaria (Sánchez y Boix, 2008).

En este estudio se pone de manifiesto que los contenidos del Máster y la formación adquirida en el mismo guardan una estrecha relación con los conocimientos adquiridos para organizar la respuesta a la diversidad en las aulas. Estos datos no concuerdan con los de autores como Sánchez (2007), en los que se manifiesta una inadecuada formación inicial del docente. De aquí se deduce que el Máster contiene elementos que nos permiten ser esperanzadores en relación con una formación de más calidad en la atención a la diversidad.

A este hecho señalado anteriormente se une que de forma mayoritaria se considere la formación a la diversidad como muy importante en la futura práctica docente, y el alumnado se sienta preparado tanto por los conocimientos adquiridos, como por la mejora en su capacidad para ser docente, así como la sensibilidad hacia la diversidad. Datos que concuerdan parcialmente con los ya expuestos por Boix (2008) y Reoyo, Carbonero, Freitas y Valdivieso (2012).

Finalmente, hay que destacar el hecho de que el profesorado participante en el Máster se sienta satisfecho con los conocimientos adquiridos sobre atención a la diversidad por su influencia en su práctica docente. Sin embargo, no se constata una influencia significativa entre la edad, la titulación de acceso y la especialidad con la percepción que tienen los docentes hacia los alumnos con necesidades específicas de apoyo educativo, lo que muestra, una vez más, un buen nivel de comprensión del futuro profesorado de Secundaria del hecho diferencial, la diversidad y la escuela inclusiva. 


\section{Referencias bibliográficas}

AGUERRONDO, I. (2004). Los desafíos de la política educativa relativos a las reformas de la formación docente. En AA.VV. Maestros en América Latina: Nuevas perspectivas sobre su formación y desempeño (pp. 97-142). Santiago de Chile: PREAL-CINDE.

BENARROCH, A. (2011). Diseño y desarrollo del Máster en profesorado de Educación Secundaria durante su primer año de implantación. Revista Eureka sobre Enseñanza y Divulgación de las Ciencias, 8(1), 20-40.

BENSO, M.C. y PEREIRA, M.C (Eds.) (2003). El profesorado de enseñanza secundaria. Retos ante el nuevo milenio. Orense: Concello de Ourense/Fundación Santa María/Universidad de Vigo.

BIRTA-SZKELY, N. (2006). Training teachers for inclusive education in Romania. Disponible en: http://www.inclues.org/english/doc/rigarep2005/Training\%20Teachers\%20for\%20I nclusive\%20Education\%20in\%20Romania_Noemi\%20Birta-Szekely.pdf (consultado el 7 de Junio de 2013).

BOLÍVAR, A. (2007). La formación inicial del profesorado de secundaria y su identidad profesional. Estudios sobre Educación, 12, 13-30.

BOIX, J.L. (2008). Nuevo profesorado de educación secundaria para nuevas realidades. Formación inicial y período umbra. REIRE. Revista d'Innovació $i$ Recerca en Educació, 1, 30-50.

BUENDÍA, L., BERROCAL, E., OLMEDO, E., PEGALAJAR, M., RUIZ, M. y TOMÉ, M. (2011). Valoración por parte del alumnado de las competencias que se pretenden conseguir con el Máster Universitario de Profesorado en Educación Secundaria Obligatoria, Bachillerato, Formación Profesional y Enseñanza de Idiomas. Bordón. Revista de Pedagogía, 63(3), 57-74.

CALVO, A. y SUSINOS, T. (2010). Prácticas de investigación que escuchan la voz del alumnado: mejorar la universidad indagando la experiencia. Profesorado. Revista de Curriculum y formación del profesorado, 14(3), 1-14.

CAMACHO, H.M. y PADRÓN, M. (2005). Necesidades formativas para afrontar la profesión docente. Percepciones del alumnado. Revista Electrónica Interuniversitaria de Formación del Profesorado, 8(2), 1-7.

CONKLIN, H. (2012). Tracing learning from divergent teacher education pathways into practice in middle grades classrooms. Journal of Teacher Education, 63(3), 171-184.

CONTRERAS, J. (2010). Ser y saber en la formación didáctica del profesorado: una visión personal. Revista Interuniversitaria de Formación del Profesorado, 68(24,2), 61-82.

EURYDICE (2008). Autonomía y responsabilidades del profesorado en Europa. Bruselas: EURYDICE. 
FORTEZA, D. (2011). Algunas claves para repensar la formación del profesorado sobre la base de la inclusión. Revista Interuniversitaria de Formación del Profesorado, 70(25,1), 127-144.

FULlAN, M. (2002). Las fuerzas del cambio. Explorando las profundidades de la reforma educativa. Madrid: Akal.

GONZÁLEZ, J.C., JIMÉNEZ, J.R. y PÉREZ, H.M. (2011). El nuevo modelo formativo del profesorado de Educación Secundaria y su proceso de implantación en las universidades andaluzas. Revista Fuentes, 11, 67-85.

GONZÁLEZ SANMAMED, M. (2009). Una nueva oportunidad para la formación inicial del profesorado de Educación Secundaria. Revista de Educación, 350, 57-78.

HARGREAVES, A. (2005). Profesorado, cultura y postmodernidad: cambian los tiempos, cambia el profesorado. Madrid: Ediciones Morata.

HERNÁNDEZ, Maj. y CARRASCO, V. (2012). Percepciones de los estudiantes del Máster en Formación del Profesorado de Educación Secundaria: fortalezas y debilidades del nuevo modelo formativo. Enseñanza \& Teaching, 30(2), 127-152.

IMBERNÓN, F. (2007). La formación inicial del profesorado de secundaria, ¿sigue siendo un tema pendiente? Aula de Innovación Educativa, 161, 5-6.

KORTHAGEN, F.A. (2010). La práctica, la teoría y la persona en la formación del profesorado. Revista Interuniversitaria de Formación del Profesorado, 68(24,2), 83-102.

LEY ORGÁNICA 2/2006 de 3 de Mayo de Educación (B.O.E. 04-05-2006).

PÉREZ, A.I. (2010). Aprender a educar. Nuevos desafíos para la formación de docentes. Revista Interuniversitaria de Formación del Profesorado, 68(24,2), 3760 .

PUELLES, M. (coord.) (2009). Profesión y vocación docente: presente y futuro. Madrid: Biblioteca Nueva.

REOYO, N., CARBONERO, M.A., FREITAS, A. y VALDIVIESO, J.A. (2012). La percepción de los futuros profesores sobre los docentes de Educación Secundaria. International Journal of Developmental and Educational Psychology INFAD Revista de Psicología, 1(2), 389-396.

RECCHIA, S. y PUIG, V. (2011). Challenges and inspirations: Students teacher's experiences in early childhood special education classrooms. Teacher Education and Special Education, 34(2), 133-151.

RODRIGUES, M.T. y ARANTES, V. (2007). La investigación como estrategia pedagógica en el proceso de formación de educadores: Relato de práctica Docente. Profesorado. Revista de currículum y formación del profesorado, 1(1), 1-14.

SÁNCHEZ, A. (2007). Investigación sobre la formación inicial del profesorado de educación secundaria para la atención educativa a los estudiantes con necesidades 
educativas. Revista Interuniversitaria de formación del profesorado, 21(2), 149181.

SÁNCHEZ, A. y BOIX, J.L. (2008). Los futuros profesores de educación secundaria: inicio de su profesionalización y construcción de su identidad docente. Revista Electrónica Interuniversitaria de Formación del profesorado, 11(2), 31-45.

SANTOS, M.A. (2010). La formación del profesorado en las instituciones que aprenden. Revista Interuniversitaria de Formación del Profesorado, 68(24,2), 175200.

SYKES, G., BIRD, T. y KENNEDY, M. (2010). Teacher education: Its problems and some prospects. Journal of Teacher Education, 61, 464-476.

TIMOSTSUK, I. y UGASTE, A. (2010). Student Teachers'Professional Identity. Teaching and teacher Education, 26(8), 1563-1570.

TRIBÓ, G. (2008). El nuevo perfil profesional de los profesores de secundaria. Educación XXI, 11, 183-209.

VEZUB, L. (2007). La formación y el desarrollo profesional docente frente a los nuevos desafíos de la escolaridad. Profesorado. Revista de currículum y formación del profesorado, 11(1), 1-23.

VAILLANT, D. (2009). Formación de profesores de Educación Secundaria: realidades y discursos. Revista de Educación, 350, 105-122.

VELA, J.A. (2010). Grados, la futura formación del docente y la atención a la diversidad. En T. Susinos (dir.). La educación inclusiva hoy: escenarios y protagonistas. Actas del Congreso Internacional y XXVIII Jornadas Nacionales de Universidades y Educación Especial (pp. 762-779). Santander: Universidad de Cantabria.

VIGO, B., SORIANO, J. y JULVE, C. (2010). Preparando profesores para la atención a la diversidad: potencialidades y limitaciones de un proyecto de innovación y mejora interdisciplinar. Revista Interuniversitaria de Formación del Profesorado, 69(24,3), 147-165.

VILCHES, A. y GIL-PÉREZ, D. (2010). Máster de formación inicial del profesorado de Enseñanza Secundaria. Algunos análisis y propuestas. Revista Eureka sobre Enseñanza y Divulgación de las Ciencias, 7(3), 661-666.

\section{Correspondencia con los autores}

$\mathrm{M}^{\mathrm{a}}$ Jesús COLMENERO RUIZ

Universidad de Jaén

Paraje "Las Lagunillas, s/n.

Teléfono: 953211803

e-mail:mjruiz@ujaen.es 